\title{
Dependencia espacial de la tasa municipal de desempleo en Andalucía. Un análisis espacial exploratorio
}

\author{
Spatial dependence of the municipal rate of unemployment in \\ Andalusia. An exploratory spatial data analysis
}

\author{
Antonio Jesús Acevedo Blanco \\ Universidad Nacional de Educación a Distancia \\ aacevedo13@alumno.uned.es(ESPAÑA) \\ Violante Martínez Quintana \\ Universidad Nacional de Educación a Distancia \\ vmartin@poli.uned.es (ESPAÑA)
}

Recibido: 27.042020

Aceptado: 03.05.2021

\section{RESUMEN}

El presente trabajo examina la dependencia espacial, global y local, de la Tasa Municipal de Desempleo en Andalucía. Aplicando el software Geoda realizamos en primer lugar un ejercicio exploratorio encaminado a identificar los valores atípicos superiores del indicador. Posteriormente se implementan estadísticos propios del Análisis Espacial Exploratorio para determinar la dependencia espacial del desempleo en Andalucía. En la última sección de resultados se identifican los puntos calientes (Hot Spots) de mayor incidencia en la construcción del indicador de autocorrelación espacial. Se concluye tras el examen de resultados en la necesidad de añadir el territorio entre las variables de análisis para el estudio sociológico del paro.

\section{PALABRAS CLAVE}

Desempleo, Andalucía, ESDA, Geoda, I de Moran. Dependencia Espacial. 


\begin{abstract}
The current paper examines the spatial, global and local autocorrelation of the Municipal Unemployment Rate in Andalusia. Applying the Geoda software, firstly, we carried out an exploratory exercise aimed at identifying the superior outliers of the indicator. Later, statistics of the Exploratory Spatial Data Analysis are implemented to determine the spatial dependence of unemployment in Andalusia. In the last section of results, the hot spots with the highest incidence in the construction of the spatial autocorrelation indicator are identified. After examining the results it's concluded on the need to add the spatial context among the analysis variables for the sociological research of unemployment.
\end{abstract}

\title{
KEY WORDS
}

Unemployment, Andalusia, ESDA, Geoda, Moran's I. Spatial Dependence.

\section{INTRODUCCIÓN}

"Data of geographic units are tied together, like bunches of grapes, not separate, like balls in an urn" (Stephan, 1934:165).

Sociología, estadística y desempleo son disciplina, herramienta y concepto interrelacionados y mutuamente constructivos desde el inicio del paro como categoría social, de la Sociología como disciplina académica y de la estadística como instrumento y técnica de análisis empírico en las sociedades industriales (Gómez-Garrido, 2005). Así metodologías y herramientas estadísticas en combinación con procedimientos y técnicas cualitativas han determinado el paradigma analítico que la Sociología ha utilizado habitualmente para explicar el paro.

Tanto nacional como internacionalmente la ciencia social ha enfocado su atención en tipologías individuales para abordar su análisis. En concreto esta taxonomía gira en torno a tres categorías o tipologías construidas según la edad de las personas: mayores o parados de larga duración, mujeres y jóvenes. Así los parados de larga duración, normalmente personas mayores hasta el estallido de la crisis de 2008, han sido la cohorte objeto de estudio para Martínez-Quintana (2004, 2011) que obtiene que las personas más vulnerables provienen de sectores muy tradicionales, antiguas ocupaciones, niveles educativos muy bajos, y de regiones de escaso dinamismo para generar empleo. Despuntan tres grupos afectados: minusválidos, mujeres mayores y personas de menor cualificación, y padecen desigualdades, vulnerabilidad, estigmatización y desánimo personal. Estas y otras realidades del paro de larga duración también fueron analizadas ampliamente por Santos-Ortega (2004) en su tesis doctoral. De igual forma la juventud y el desempleo han dado cuerpo a un campo de estudios amplio y relevante abordado por autores como Alonso, Rodríguez, y Rojo (2017), o Stamm, 
(2006) . Por último, el desempleo femenino ha sido una de las variables principales de análisis desde las primeras aproximaciones a la temática del género y el paro propuestas por Jahoda (1987). Así Poveda-Rosa (2006) o Martínez-Tola y de la Cal (2017) también han abordado en sus trabajos la componente de género en los estudios del desempleo.

Consecuentemente la Sociología del Paro ha enfocado su análisis en torno a componentes psicosociales de corte individual que de una forma u otra tratan de explicar los interrogantes siguientes: qué sienten los parados, cómo piensan, qué salud tienen o desde el enfoque de Sanchis-Gómez ( 2017) "¿Por qué no se revelan?".Sin embargo, la premisa territorial y/o espacial del contexto de hábitat residencial del desempleo no ha tenido la misma atención en Sociología que otras variables de perfil individual. De aquí que el presente artículo se vuelque en la utilidad que pueda tener el análisis espacial exploratorio vinculado a la Sociología del Paro, con el fin de poder obtener unos mapas territoriales y poder trabajar desde ahí los procesos de interacción social, político y laboral de las realidades que presenta el paro en el territorio geográfico de residencia de la población de desempleados andaluces.

Por lo tanto, se redefinen los conceptos desde una perspectiva macrosociológica y se aplican los métodos exploratorios de análisis y minería de datos espaciales orientados, entre otros, a dilucidar si existe patrón de dependencia espacial en la Tasa Municipal de Desempleo en Andalucía (TMD) ${ }^{1}$, y si este análisis pudiera ser útil para comprender la correlación entre el territorio y las tasas de paro en los municipios de Andalucía. Para este objetivo proponemos un ejercicio espacial exploratorio de la TMD centrado en presentar su distribución geográfica en el territorio, identificar valores atípicos en los datos y localizar clústeres o agrupaciones de municipios con alta incidencia de desempleo.

El avance de los Sistemas de Información Geográfica (SIG) (GutiérrezPuebla y García-Palomares, 2016) unido a la cada vez mayor disponibilidad de datos de paro municipalmente geo-referenciados posibilitan que el análisis espacial exploratorio (ESDA) ${ }^{2}$ se establezca como una herramienta de gran utilidad para la analítica del desempleo y su significación sociológica. La perspectiva teórica y analítica de este trabajo surge de considerar la causa geográfica como una tipología más dentro de las variables básicas de estudio del desempleo. Desde este ángulo se considera relevante estudiar si existe patrón de autocorrelación espacial del paro en Andalucía por dos motivos fundamentales. Por una parte, desde el punto de partida de una explicación sociológica que atiende al territorio como variable explicativa de los fenómenos sociales. En otras palabras, se plantea considerar el factor geográfico, municipio de residencia del desempleado en nuestro caso, como un elemento más, diferenciador y causal, en las situaciones de paro. Por otra parte, desde un enfoque econométrico la autocorrelación entre indicador y espacio geográfico puede plantear problemas de especificación en las

\footnotetext{
${ }^{1}$ Propuesta por el Instituto de Estadística y Cartografía de Andalucía (IECA)

2 Exploratory Spatial Data Analysis (ESDA). Utilizamos en el artículo siglas en inglés para facilitar búsquedas bibliográficas.
} 
regresiones utilizadas para la estadística del desempleo reduciendo considerablemente su validez en caso de confirmarse patrones de dependencia espacial (DE).

\section{REVISIÓN DEL MARCO TEÓRICO}

Se observa por varios autores que la premisa sociedad y espacio geográfico-social son mutuamente constructivos; es decir, que las características de las personas, las sociedades y el espacio geográfico donde se desarrolla la vida real se encuentran correlacionadas entre sí (Soja, 1980). Los hechos sociales están localizados en el espacio y en el tiempo, así como que toda agencia social está delimitada y rodeada de un contexto que lo interconecta con el espacio físico y con el tiempo (Therborn, 2006). Por tanto el espacio geográfico importa, influye en los comportamientos, identidades y formas de entender el mundo de las colectividades resultando necesaria su inclusión en los modelos explicativos de conocimiento de las ciencias sociales (Agnew, 1996). Se trata pues de abordar parte de la perspectiva sociológica clásica, ya puesta de relieve por Durkheim, (1992) en su estudio del suicidio donde argumentaba empíricamente la dependencia del hecho social con el contexto geográfico. Esta relación entre hecho social y contexto territorial ha sido una constante en el paradigma sociológico en la búsqueda de las explicaciones y de significación social. En palabras de Porter (2011) que alude a Mills (1977), las dinámicas de producción académica actuales plantean cada vez más y en mayor medida la emergencia de una "GeoSociological Imagination" en la que perspectivas de análisis, métodos y marcos conceptuales se proponen orientados a extender caminos de convergencia interdisciplinarios hacia un paradigma en ciencia social "espacialmente integrado" (Rodrigues-Silveira, 2013).

Por consiguiente, para comprender las relaciones entre el nivel contextual y el desempleo andaluz podría ser de utilidad no tratar a las colectividades de individuos residentes en unidades espaciales (municipios) como "islas independientes" no influenciadas por las características sociolaborales de las unidades espaciales vecinas. Este paradigma Geo-Sociológico de interconexión entre fenómenos y geografía social surge a partir del punto de partida seminal sugerido por Waldo Tobler en la primera Ley de la Geografía o principio de autocorrelación espacial. La proposición plantea la similitud de características entre vecinos con respecto a los fenómenos sociales pues: "todas las cosas están relacionadas entre sí, pero las cosas más próximas en el espacio tienen una relación mayor que las distantes" (Tobler, 1970:234).

Desde esta perspectiva Sui propone diseccionar en dos partes este principio axiomático. En primer lugar, si en el espacio geográfico "todas las cosas están relacionadas entre sî̀' obviamente se pone de relieve que existen patrones de interrelación entre fenómenos sociales y espacio físico. Por otra parte, si "las cosas cercanas están más relacionadas que las distantes" implica que existen factores y circunstancias locales que pueden hacer que una ubicación sea significativamente diferente de otras. Si la primera propuesta denota explícitamente la depen- 
dencia espacial (DE) de los fenómenos sociales, la segunda declaración connota implícitamente heterogeneidad espacial (HE) (Sui, 2004). La existencia en los datos geográficamente referenciados de DE y HE condicionan la necesidad del análisis local por la simple lógica de que factores sociológicos, culturales, actitudes, comportamientos velados o la incidencia de políticas públicas focalizadas puedan configurar contextos heterogéneos en la distribución territorial de los indicadores.

Siguiendo a Chasco (2004) cuando se trabaja con unidades espaciales (países, naciones, comunidades autónomas, municipios, secciones censales) en las que los fenómenos sociales puedan distribuirse por un patrón de similitud de características entre vecinos, puede darse la posibilidad de que surjan modelos locales de $\mathrm{HE}$, o, en otros términos, un patrón de comportamiento de los datos condicionado por su ubicación en el espacio geográfico. La HE suele darse en contextos de polarización de indicadores del tipo norte/sur, sector industrial/ sector servicios o barrios residenciales/suburbios. Así pues, ante la duda de HE se hace necesario investigar en torno a la DE entre variables y el contexto geográfico.

El punto de partida para considerar pertinente el uso de ESDA en el estudio del desempleo surge cuando los valores de la variable dependiente en una unidad espacial pueda ser función del valor de la misma variable en unidades vecinas. De aquí que en este trabajo la posible existencia de autocorrelación espacial se traduce en determinar si la TMD correlaciona con las TMD de los municipios vecinos formando territorios de similar incidencia de desempleo. Es en estos casos y ante la evidencia de existencia de HE cuando se pone de manifiesto la validez del contexto geográfico en la definición de los fenómenos sociales (Chasco, 2003).

La HE suele presentarse en las variables espacialmente intensivas debido a una "inestabilidad estructural en forma de varianza no constante de los residuos de una regresión (heteroscedasticidad) o en los coeficientes del modelo" (Anselin, 2007:4). Acevedo-Bohórquez y Velásquez (2008) apuntan que la HE consiste en ausencia de estabilidad en la dispersión geográfica de un fenómeno, por tanto, esta inestabilidad en los residuos de la regresión debido a la posible DE puede sugerir una contrariedad estadística que, en caso de no ser tenido en cuenta, llegue a plantear problemas de especificación (Vilalta, 2005).

DE se define como la existencia de una relación entre lo que sucede en un punto del plano y lo que sucede en otro lugar (Anselin y Bera, 1998). La dependencia espacial es también conocida como "autocorrelación espacial" (Anselin y Rey, 1991) aunque la noción de autocorrelación espacial es más limitada que la dependencia espacial en la práctica ambos conceptos generalmente se consideran sinónimos. Sin embargo, en el campo de la estadística espacial se utiliza principalmente el término "Dependencia Espacial".

Para la literatura económica la DE en las tasas de desempleo locales es una temática de creciente interés. Möller y Soltwedel (2007) proponen una revisión sistemática de contribuciones sobre esta temática en la que el nivel de desagregación territorial es el aspecto central. Entre otros, resultan especialmente 
relevantes las contribuciones de Patacchini y Zenou, (2007) con su trabajo orientado a explicar la autocorrelación del desempleo en "Travel to Work Areas (TTWAs)" del Reino Unido, López-Hernández (2013) que propone examinar la ratio de desempleo mediante una matriz de distancias de segundo orden para los municipios andaluces de más de 1000 habitantes, Prodromídis (2012) que estudia la dependencia espacial del desempleo femenino en municipios griegos, Netrdová y Nosek (2016) que focalizan el análisis espacial en el nivel municipal conjunto para Chequia, Austria, Alemania y Polonia, Netrdová y Nosek (2020) con matrices espaciales de distancia analizan la evolución del desempleo checo a nivel municipal o Kosfeld y Dreger que en artículos de (2006) y (2019) centran su investigación en capturar la dependencia espacial del desempleo y el empleo en las áreas de trabajo alemanas.

En España son varios los estudios que analizan la DE del desempleo. Así Alonso-Villar, del-Río, y Toharia (2009) examinan la distribución espacial del desempleo a distintos niveles de desagregación. Igualmente López-Bazo, Del Barrio y Artís (2002) dan cuenta de la polarización de paro y posteriormente en (2005) examinan la correlación espacial del desempleo en la década 19902000, ambos trabajos se plantean con un nivel de desagregación provincial. En la perspectiva del servicio al desempleado Suárez, Mayor, y Cueto (2012) revisan la cuestión de la oferta de empleo de los servicios públicos en el nivel regional. Más recientemente las aportación de Rodríguez Moya y Pozo Rivera (2019) proponen, conjuntamente con el análisis de otras variables socio-económicas, explorar la DE del desempleo para la Comunidad de Madrid desagregada municipalmente. Desde el mismo enfoque Manzanares-Gutiérrez et al., (2016) y Manzanares-Gutiérrez y Riquelme-Perea (2017) ponen el énfasis en los Mercados de Trabajo Locales (MTL) tanto del conjunto del estado español, como de la provincia de Huelva. En este último caso resulta especialmente relevante que para testar la DE se utilizan diferentes matrices de pesos espaciales, perspectiva de análisis adoptada en este trabajo.

La contribución original que se propone en esta investigación radica principalmente en el indicador y en la base cartográfica. En primer término y con respecto al indicador, la TMD se aporta un nivel de desagregación municipal en el estudio del desempleo aún poco explorado. Cabe destacar que los datos se procesan a partir de registros tanto de Afiliaciones a la Seguridad Social como de Demandantes de Empleo No Ocupados (DENOS). Por otro lado, reseñar que el Instituto de Estadística y Cartografía de Andalucía (IECA) ha puesto en marcha de un tiempo a esta parte toda una serie de recursos denominados Datos Espaciales de Referencia de Andalucía (DERA) que constituyen un repertorio de bases cartográficas de diferente naturaleza presentada en bloques temáticos que permiten el acceso centralizado y gratuito a investigadores, profesionales, docentes y técnicos.

Unido a la publicación trimestral de los datos necesarios para desarrollar la TMD la base cartográfica de este artículo abarca la totalidad de los municipios andaluces. Si bien desde una perspectiva estrictamente metodológica trabajar con la totalidad de municipios andaluces (786 municipios) condiciona el análisis 
al aumentar las posibilidades de HE, cabe destacar que la actualización constante de los datos por parte del IECA facilita un marco de observación con amplias posibilidades prácticas de reajuste de los datos, factor esencial para una analítica ESDA que puede ser de utilidad en el estudio del desemple.

\section{MÉTODO Y PROCEDIMIENTOS}

El método de análisis ESDA está esencialmente indicado en las fases previas de modelización econométrica espacial o, a modo exploratorio, cuando no existe un marco teórico previo suficientemente desarrollado acerca del fenómeno que se pretende explicar. Estas situaciones se plantean muy a menudo en ciencias sociales debido al carácter contingente de los fenómenos sociales y la multidisciplinariedad metodológica existente para abordarlos. La principal fortaleza de ESDA se basa en su capacidad de minería de datos (Dall'erba, 2009) enfocada al análisis de grandes bases de datos geográficos cuya distribución espacial no tiene por qué ser conocida a priori. Luc Anselin (1999:68) define ESDA como "conjunto de técnicas que describen y visualizan distribuciones espaciales, identifican localizaciones atípicas ("spatial outliers"), descubren esquemas de agrupación espacial, asociaciones ("clusters") o puntos calientes ("hot spots") y sugieren estructuras espaciales u otras formas de dependencia”. En este sentido ESDA sugiere diseños de investigación inductivos en los que las variables de análisis se les confiere "(...) un carácter descriptivo (estadístico) más que confirmatorio (econométrico)” (Chasco, 2003b:30).

\subsection{Base cartográfica y software.}

Para este artículo hemos utilizamos la base cartográfica que proporciona el IECA $^{3}$ en la sección de Datos Espaciales de Referencia para Andalucía (DERA). La capa relativa a límites de términos municipales (13_01_Termino_municipal) contiene la delimitación geográfica de los municipios andaluces. El registro incorpora los archivos y extensiones necesarias para el tratamiento informático. Por su parte el software libre utilizado para este artículo, GeoDa, presenta un marco de trabajo de entorno amigable e interactivo con un enfoque que propone ir más allá del simple mapeo de variables orientando los procesos a traducir grandes conjuntos de datos, geográficamente referenciados, en patrones espaciales de comportamiento de las variables (Li, Anselin, y Koschinsky, 2015).

\footnotetext{
${ }^{3}$ https://www.juntadeandalucia.es/institutodeestadisticaycartografia/DERA/
} 


\subsection{Variable espacialmente intensiva. Tasa Municipal de Desempleo.}

La Tasa Municipal de Desempleo (TMD) $)^{4}$, desarrollada por el IECA ${ }^{5}$ propone un indicador de desempleo cociente de adicionar en el denominador el registro de "Demandantes de Empleo no Ocupados" (DENOS) los "Afiliados a la Seguridad Social en alta laboral que trabajan en Andalucía" (ASSrm). El numerador resulta del registro DENOS. Denominador y numerador se presentan desagregados municipalmente representado el primero una aproximación a la población activa municipal. El cálculo de la tasa refleja la media anual de los registros por municipio del año 2019.

\section{TMD $=$ DENOS $/($ DENOS + ASSrm $)$}

El registro DENOS, a diferencia del registro de agregados "Paro Registrado", refleja situaciones de desempleo más amplias. DENOS "se calcula sumando al colectivo de parados registrados a otros grupos que presumiblemente serían considerados parados si se aplicaran los criterios internacionales en los que se basan las cifras de la Encuesta de Población Activa (EPA)" (Albert y Toharia, 2007:15). La Orden de 11 de marzo de $1985^{6}$ por la que se establecen criterios estadísticos para la medición del paro registrado excluye del paro registrado colectivos como Trabajadores Eventuales Agrarios Subsidiados (TEAS), estudiantes, demandantes de empleo de duración inferior a tres meses o demandantes de empleo de jornada inferior a las veinte horas. De los colectivos reseñados es el de TEAS, solo existente en Andalucía y Extremadura, el agregado que en mayor medida puede sesgar el análisis con su exclusión debido al gran peso de esta población en los municipios agrarios andaluces.

El agregado ASSrm resulta de la explotación estadística que realiza el IECA $^{7}$ a nivel municipal. Para el producto ASSrm se recopilan los ficheros de microdatos del registro de afiliaciones a la Seguridad Social y el archivo de Cuentas de Cotización en Andalucía. De forma trimestral el IECA somete a cruces y depuración ambos registros juntos al nomenclátor municipal del Instituto Nacional de Estadística (INE) con el objetivo de mantener actualizado la codificación de los municipios andaluces. Se publican datos tanto de afiliaciones, como de afiliados, dado que una misma persona puede tener más de una afiliación. Esta peculiaridad de la explotación estadística resulta determinante pues posibilita la investigación del desempleo desagregado municipalmente al eliminar las situaciones de pluriafiliación.

${ }^{4}$ DataSet (Acevedo-Blanco 2021). En el capítulo de referencias se localiza link a la base de datos. Contiene este data-set los ficheros necesarios para tratamiento con GeoDa. Descarga libre que permite referenciar, replicar y/o ampliar este trabajo.

${ }_{5}^{5}$ https://www.juntadeandalucia.es/institutodeestadisticaycartografia/sima/info.htm?f=j11

6 https://www.boe.es/buscar/doc.php?id=BOE-A-1985-4112

7 http://www.juntadeandalucia.es/institutodeestadisticaycartografia/merctrab/metodologia/ MT040102.pdf 
Aunque en ciencia social debemos de tener presente que la relación entre indicador y dimensiones de estudio son siempre parciales, la tasa municipal de desempleo plantea sus principales fortalezas explicativas en torno a:

- Facilitar la comparabilidad en materia de desempleo entre municipios.

- Facilitar el recuento de parados con cifras totales desagregadas municipalmente ofreciendo datos de los mercados laborales locales con un nivel de desagregación no abordado por la EPA.

- Al ser una media anual da cuenta de una mayor variabilidad de situaciones sociales ligadas al paro, precariedad y eventualidad.

- Aunque la tasa la presentamos anual la explotación estadística del IECA permite tanto la clasificación trimestral como por sexo y tramos de edad.

- Se facilita el análisis por provincias, comarcas y entes locales, permitiendo la máxima desagregación posible en el estudio del desempleo.

Por el contrario, la principal debilidad de la tasa de desempleo deriva de la imposibilidad de explicitar con el indicador una teoría de la población subyacente. La unidad de análisis es el municipio, por lo que la tasa compara al mismo nivel municipios con una densidad poblacional alta como Almería, Málaga, Granada o Jerez con otros cuya densidad poblacional es sensiblemente menor ${ }^{8}$.

\subsection{Dependencia global, el estadístico I de Moran.}

El estadístico de prueba I Moran (Moran, 1950) es en pocas palabras un coeficiente de correlación entre una variable espacialmente intensiva y su retardo espacial. El retardo espacial consiste en un promedio ponderado de los valores de una variable en las localizaciones vecinas a una dada. En otras palabras, el valor espacialmente retardado de una variable en una localización i corresponde a la suma ponderada de todos los valores de dicha variable en las localizaciones vecinas a i. El estadístico I de Moran permite conocer el grado de autocorrelación de una variable, es decir, hasta qué punto existe alguna tendencia o concentración de valores de una variable en determinadas zonas de la geografía y su grado de correlación con los valores de las ubicaciones vecinas (Chasco y Fernández-Avilés, 2009).

Al igual que el coeficiente de correlación de Pearson el estadístico I de Moran expresa una medida de autocorrelación espacial con valores que van desde -1 a 1 donde el valor 1 indica correlación perfecta, 0 ausencia de correlación y -1 dispersión perfecta. El valor del estadístico I de Moran puede utilizarse igualmente, bien como indicador del ajuste de la nube de puntos de la regresión entre la variable espacialmente intensiva y su retardo, o bien, para la detección de los

\footnotetext{
${ }^{8}$ Alternativamente el programa GeoDa permite entre otras funcionalidades la construcción de ratios con enfoque empírico bayesiano, esquema especialmente indicado para suavizado de tasas poblacionales. Los resultados obtenidos que aplican este enfoque no varían sustancialmente de los ofrecidos por la tasa bruta. Se decidió para este trabajo optar por la TMD sin transformaciones.
} 
valores atípicos y residuos inestables. Indudablemente este estadístico se verá afectado por aquellos puntos atípicos sensiblemente divergentes de la media de la variable.

El procedimiento comienza con el usuario introduciendo una matriz de contigüidad para identificar los objetos espaciales. Esta matriz, siguiendo diferentes criterios de adyacencia operacionaliza la adscripción a la categoría de vecino de una ubicación dada. La conectividad que define la vecindad se incorpora en la matriz "W" que representa a los objetos espaciales en cada fila i. A su vez la matriz contiene elementos correspondientes a las mismas ubicaciones representadas igualmente en las columnas $\mathrm{j}$. La estructura de $\mathrm{W}_{\mathrm{ij}}$ identifica las ubicaciones vecinas a una dada de forma que los valores 1 representan "vecinos" y los valores 0 implican no vecindad.

$$
W=\left(\begin{array}{cccc}
w_{11} & w_{12} & \ldots & w_{1 n} \\
w_{21} & w_{22} & \ldots & w_{2 n} \\
\vdots & \vdots & \ddots & \vdots \\
w_{n 1} & w_{n 2} & \ldots & w_{n n}
\end{array}\right)
$$

La forma del índice de Moran para cada ubicación $i$ es la siguiente:

$$
I_{i}=\left(z_{i} / m_{2}\right) \sum_{j=1}^{n} w_{i j} z_{j}
$$

donde $m=$ varianza y las observaciones $z$ desviaciones de la media $Z=\left(X_{i}-\overline{\bar{X}}\right)$. El sumatorio de todos los valores locales de la I de Moran conforma el índice I de Moran global (Celemín, 2009).

$$
\sum_{i=1} I_{i}=\sum_{i=1}^{n} z_{i} \sum_{j=1}^{n} w_{i j} z_{j}
$$

El estadístico I de Moran se utiliza como una medida de dependencia espacial y se evalúa similar al procedimiento para el contraste hipótesis nula ${ }^{9}$ (Almazán et al., 2011). La validez se prueba en base a una distribución de referencia obtenida permutando aleatoriamente los valores observados. La prueba de alea-

${ }^{9} \mathrm{H}_{0}=$ datos distribuidos al azar por el mapa / $\mathrm{H}_{1}=$ DE en la variable. 
toriedad parte de la proposición de entender que sí sobre un objeto espacial de $n$ valores, $n$ ! permutaciones de los datos son posibles. Los valores I de Moran esperados obtenidos en estas permutaciones se representan gráficamente en Geoda en un histograma que nos permiten varias pruebas para comprobar la hipótesis de aleatoriedad de la variable (Celemín, 2009):

- Valor de I positivo y elevado que invita a considerar que existe patrón de autocorrelación espacial de la variable, por tanto, los valores de la variable "dependen" y pueden ser explicados en gran medida por los valores de las ubicaciones vecinas.

- $\quad$ Valor p. Cuando las pruebas han tenido un solo valor positivo de las 999 permutaciones realizadas, por tanto, el resultado es: $1 /(999+1)$ $=0.001$. En este caso el p-valor es de 0.001, menor a 0.05 nos lleva a plantear rechazo de hipótesis nula.

- Valor z. Cuando los valores obtenidos por permutación (z_value) son lo suficientemente elevados para que, al aproximarlos a valores de corte $\mathrm{z}$ en distribuciones normales, se sugiera el rechazo la hipótesis nula.

\subsection{Dependencia local, el estadístico I local de Moran.}

Cada unidad espacial debido a su ubicación relativa en el espacio geográfico y su posible comportamiento socialmente diferenciado posee un grado intrínseco de particularidad especifica propia. En el análisis local debido a la HE las ubicaciones espaciales revelan tanto la intensidad en los valores extremos de la variable, como los patrones de DE que cada objeto espacial posee en relación el retardo espacial.

La DE global que puede revelar el global I de Moran no detecta la significatividad de las ubicaciones locales. Para este fin (Anselin, 1995) desarrolla el algoritmo I local de Moran al igual que su representación gráfica o mapa LISA (Local Indicators of Spatial Association). El algoritmo $I_{i}$ calcula un estadístico por cada una de las ubicaciones espaciales para comprobar si en cada unidad i existe una asociación significativa de valores análogos (altos o bajos) de la siguiente forma:

$$
I_{i}=\frac{\dot{y}_{l}}{m_{2}} \sum_{j \in J_{i}} W_{I J} \dot{y}_{J}
$$

Siendo $\dot{y}_{l}$ e $\dot{y}_{j}$ los valores de la variable $Y$ tomados en desviaciones de la media en las ubicaciones $i, j$. $W_{i j}$ hace referencia a la matriz de pesos espaciales, $m_{2}$ reseña un factor de proporcionalidad constante en todas las unidades espaciales y $J_{i}$ hace referencia al conjunto de unidades relacionadas con i. El resultado 
evidencia que la media de los valores de $I_{i}$ será igual a la media de I por el factor de proporcionalidad.

Para cada una de la ubicación se realiza un proceso de permutación condicional. El valor del indicador en una ubicación es permutado aleatoriamente conforme al valor real de la estadística I de Moran en el municipio. El proceso es repetido reiteradamente hasta obtener un margen lo suficientemente amplio (999 permutaciones) para alcanzar un pseudo valor p para cada ubicación que posteriormente puede utilizarse para evaluar la significatividad. Esta noción de significatividad no es la común y por tanto no debe interpretarse en el sentido de significatividad estadística. La significatividad se calcula en base a la contribución de cada unidad espacial para la construcción del global de Moran en función del valor que la variable toma en una de las unidades espaciales y el valor promedio de las ubicaciones vecinas (retardo espacial).

\section{RESULTADOS}

Se sigue un proceso de tres pasos para realizar el análisis:

1. Análisis exploratorio y visualización de la distribución espacial de la tasa

2. Agrupación espacial global mediante el estadístico I global de Moran con matrices de contigüidad y distancia. Pruebas permutaciones aleatorias. Regresión espaciotemporal.

3. Mapas LISA para evaluar la DE local para cada una de las matrices de pesos construidas.

4. Depurar los resultados para detectar y localizar "hot spots" (puntos calientes).

\subsection{Análisis exploratorio univariante TMD. Descriptivos y atípicos.}

En la parte inferior del gráfico de caja (Fig 1) se enumeran las estadísticas descriptivas expresadas en tanto por ciento de la TMD para el año 2019 que incluyen; el primer (17.84\%) y tercer cuartil (23.78\%), valor de rango mínimo (6.89\%) y máximo (38.71\%), media (20.98\%), mediana (20.70\%) y desviación estándar (4.92). Significar que en nuestro caso el valor de la media se sitúa en $20.98 \%$ casi coincidiendo con la mediana de la distribución, por tanto, sugiriendo una alineación simétrica de la distribución. 
Figura 1: Diagrama de caja TMD 2019.

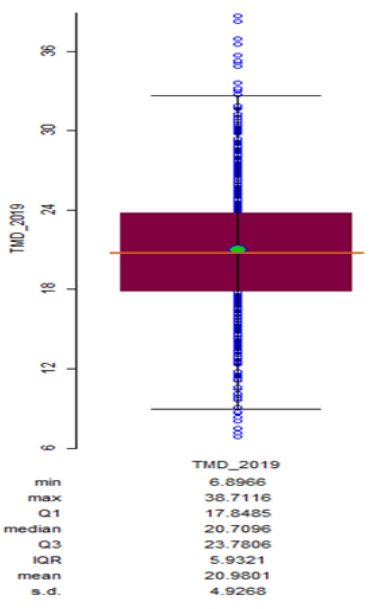

Fuente: Elaboración Propia a partir de IECA con GeoDa.

Centrándonos en los outliers superiores, trece municipios presentan valores que exceden el rango de valores comprendidos en los cuartiles de la distribución con un promedio de desempleo en este grupo del 35,50\% (Tabla 1) casi quince puntos porcentuales por encima de la media. Tres municipios pertenecen a la provincia de Cádiz, dos a la provincia de Huelva, seis a la provincia de Córdoba y uno (La Carolina) a la provincia de Jaén. Casi duplicando a la media el caso más extremo se presenta en el municipio cordobés de Bélmez con una TMD del $38.71 \%$ de desempleo ${ }^{10}$.

${ }^{10}$ Wikipedia: https://es.wikipedia.org/wiki/Belmez: "La economía de Bélmez está notablemente condicionada por su especificad geográfica, su inserción plena en la franja de depresión socioeconómica del Alto Guadiato y el déficit de infraestructuras varias que soporta, se ha unido el éxodo rural de las últimas décadas y la especialización excesiva de la economía local en el sector minero-industrial. 
Tabla 1: Atípicos superiores de la TMD

\begin{tabular}{|l|l|l|l|}
\hline Provincia & \multicolumn{1}{|c|}{ Municipio } & Código INE & TMD_2019 \\
\hline Córdoba & Bélmez & 14009 & $38,71 \%$ \\
\hline Cádiz & Barbate & 11007 & $38,24 \%$ \\
\hline Córdoba & Peñarroya-Pueblonuevo & 14052 & $36,98 \%$ \\
\hline Cádiz & Línea de Ia Concepción (La) & 11022 & $36,92 \%$ \\
\hline Córdoba & Granjuela (La) & 14032 & $36,53 \%$ \\
\hline Córdoba & Fuente la Lancha & 14028 & $35,73 \%$ \\
\hline Huelva & Cala & 21016 & $35,27 \%$ \\
\hline Cádiz & Paterna de Rivera & 11025 & $35,16 \%$ \\
\hline Cádiz & Arcos de Ia Frontera & 11006 & $34,85 \%$ \\
\hline Córdoba & Fuente Obejuna & 14029 & $33,63 \%$ \\
\hline Córdoba & Blázquez (Los) & 14011 & $33,18 \%$ \\
\hline Jaén & Carolina (La) & 23024 & $33,09 \%$ \\
\hline Huelva & Alájar & 21001 & $32,99 \%$ \\
\hline
\end{tabular}

Fuente: Elaboración Propia a partir de IECA con GeoDa.

El mapa (1) refleja la distribución de TMD dispuesta en el mapa. Así en el grupo de los valores atípicos inferiores (Lower outlier) se encuentran 8 municipios con una tasa de desempleo que se sitúa entre el 6.89\% y el $8.95 \%$. El primer cuartil, con 188 municipios, contiene tasas de desempleo municipal que oscilan entre el $8.95 \%$ y el $17.84 \%$. Con un límite superior e inferior en estos parámetros tan elevados en el primero de los cuartiles no resulta aventurado declarar el carácter estructural del desempleo a nivel municipal en Andalucía. Si prestamos atención a lo cuartiles centrales de la distribución, desde el segundo cuartil al tercer cuartil, la TMD oscila desde el $17.84 \%$ al $23.78 \%$, Unas cifras de desempleo bastante elevadas y que invitan a plantear diferencias significativas en cuanto a su distribución geográfica. Por último, el cuarto cuartil refleja valores extremos de desempleo que se mueven entre desde una TMD de $23.78 \%$ al $32.69 \%$ de paro municipal. Los atípicos superiores (Upper outlier) comienzan en el corte del $32.67 \%$ hasta la cifra más alta de la distribución que se sitúa en $38.71 \%$ del municipio cordobés de Bélmez. 


\section{Mapa 1: Mapa de caja de la TMD 2019.}



Fuente: Elaboración Propia a partir de IECA con GeoDa

La concentración geográfica de los municipios con valores altos de TMD resulta evidente agrupándose éstos en torno al valle del Río Guadalquivir, provincias de Cádiz y Córdoba, y el norte de la provincia de Huelva. Una rápida visualización de este mapa ya nos permite comenzar a intuir el comportamiento desigual de la variable en el territorio, o en términos geoespaciales, se comienza a sospechar de HE en la distribución espacial de la variable. Desde la perspectiva geográfica se entrevé que en Andalucía podemos distinguir un comportamiento del desempleo diferenciado en las zonas costeras de las zonas del interior. De igual forma el mapa permite vislumbrar una línea divisoria entre el oeste y el este andaluz. Entre otras particularidades resulta especialmente interesante que el núcleo periurbano de Sevilla parece tener una conducta particular, desigual a las tasas de los municipios más alejados de la capital andaluza.

\subsection{Dependencia espaciotemporal de la TMD.}

Los indicadores socioeconómicos geográficamente intensivos evalúan la autocorrelación que pueda llegar a darse entre índice y territorio. Modelizar el territorio se consigue utilizando diferentes criterios de vecindad que vienen a significar cuáles son las ubicaciones espaciales que comparten espacio geográfico entre sí y bajo qué criterios. Además de matrices híbridas, en econometría espacial se utilizan normalmente tres criterios para la construcción de matrices de pesos espaciales; la contigüidad, la distancia y la vecindad (vecinos más cercanos). En este trabajo se evalúa la sensibilidad de la DE del paro municipal en 
Andalucía, y se utilizarán matrices de contigüidad y de distancia, que pasamos a glosar sintéticamente:

- Matriz de Contigüidad Reina (Queen); de primer orden (Q1) y de segundo orden $(\mathrm{Q} 2)$.

Para crear esta matriz de contigüidad se establecen qué ubicaciones componen el retardo espacial en analogía a los movimientos en diagonal y horizontal de la Reina en el tablero de ajedrez. Si la matriz es de primer orden, las ubicaciones vecinas vendrán determinadas por compartir fronteras adyacentes. Si la matriz es de segundo orden, las contigüidades resultan de compartir borde común por parte de las ubicaciones adyacentes a las de primer orden. En este trabajo se incluye un cálculo integrado para la construcción del retardo (Q2) tanto de vecinos de orden superior como de orden inferior.

Esta matriz de contigüidad es la más utilizada para indicadores socioeconómicos principalmente, como en el caso de los municipios, cuando los objetos espaciales son poligonales. Como podemos observar en el cuadro (1) utilizar criterios de segundo orden modeliza un territorio con mayor número medio de ubicaciones que el retardo que se propone por contigüidad simple de primer orden. Se consigue con esta matriz de segundo orden evaluar la DE del desempleo sobre la base de criterios de retardos espaciales más próximos a las características propias de homogeneidad territorial e impacto económico de los Mercados de Trabajo Locales (MTL).

Cuadro 1: Propiedades Marices de Contigüidad.

\begin{tabular}{|l|l|l|}
\hline type & queen & queen \\
\hline symmetry & symmetric & symmetric \\
\hline order & 1 & 2 \\
\hline \# observations & 786 & 786 \\
\hline min neighbors & 1 & 3 \\
\hline max neighbors & 19 & 57 \\
\hline mean neighbors & 5.84 & 21.94 \\
\hline median neighbors & 5.00 & 21.00 \\
\hline \% non-zero & $0.74 \%$ & $2.79 \%$ \\
\hline
\end{tabular}

Fuente: Elaboración propia con GeoDa. 
- Matrices de Distancias. 6 km (D1_6km)/18 km (D2_18km)/36 km (D3_36km):

Las matrices de distancias se construyen a partir de las distancias euclidianas entre puntos de un plano. Aplicado a objeto espaciales poligonales las distancias para asignar vecindad se calculan entre los centroides de los polígonos. Adoptada al estudio de la DE del desempleo la principal de las potencialidades de estas matrices se centra en modelizar en el territorio los trayectos laborales a los puestos de trabajo. En este sentido cabe destacar que los MLT se extienden sobre espacios extensos, con tiempos de commuting que según la literatura puede alcanzar hasta una hora, por tanto, parece razonable evaluar la DE del desempleo andaluz con un umbral de distancia inversa equiparable a ese tiempo de commuting.

\section{Cuadro 2: Propiedades Matrices de Distancia.}

\begin{tabular}{|r|lll|}
\hline \multicolumn{1}{|l|}{ type } & $\begin{array}{l}\text { Threshold 6 km } \\
\text { (D1_6 km) }\end{array}$ & $\begin{array}{l}\text { threshold18 } \mathrm{km} \\
\text { (D2_18km) }\end{array}$ & $\begin{array}{l}\text { Threshold 36 km } \\
\text { (D3_36 km) }\end{array}$ \\
\hline inverse distance & true & true & true \\
\hline power & 1 & 1 & 1 \\
\hline symmetry & symmetric & symmetric & symmetric \\
\hline distance metric & Euclidean & Euclidean & Euclidean \\
\hline distance vars & centroids & centroids & centroids \\
\hline threshold value & 20127.2 & 60127.2 & 120127 \\
\hline \# observations & 786 & 786 & 786 \\
\hline min neighbors & 1 & 21 & 76 \\
\hline max neighbors & 44 & 187 & 434 \\
\hline mean neighbors & 14.58 & 99.11 & 288.92 \\
\hline median neighbors & 13.00 & 92.00 & 293.00 \\
\hline \% non-zero & $1.86 \%$ & $12.61 \%$ & $36.76 \%$ \\
\hline
\end{tabular}

Fuente: Elaboración propia con GeoDa

En la tabla (2) muestran los resultados del estadístico I de Moran para cada una de las matrices de pesos espaciales construidas. Los datos de las pruebas de permutaciones aleatorias (999) permiten el rechazo de la hipótesis nula de aleatoriedad en la distribución geográfica de la TMD, por tanto, la analítica confirma indicios altamente probables de DE global del desempleo en Andalucía. Podemos comprobar como el índice de Moran desciende conforme aplicamos matrices con mayor número de vecinos. Supuesto éste por otro lado lógico, pues inevitablemente al aumentar los criterios de vecindad aumenta la HE en la composición de los retardos espaciales. 
Tabla 2: Resultados de I de Moran TMD19 y pruebas de permutaciones aleatorias.

\begin{tabular}{|l|lll|} 
Matrices & I de Moran & P-value & Z-value \\
\hline Q1 & 0,4971 & 0,001 & 26,30 \\
Q2 & 0,3803 & 0,001 & 35,10 \\
D1_6km & 0,4495 & 0,001 & 27,69 \\
D2_18km & 0,2662 & 0,001 & 50,31 \\
D3_36km & 0,1294 & 0,001 & 52,93 \\
\hline
\end{tabular}

Fuente: Elaboración propia con GeoDa.

Normalmente la DE en las tasas de paro resultan muy estructurales, al menos eso parece justificarse tras revisar los resultados mostrados en la tabla (3). Los resultados evidencian la similitud de los valores del indicador para todos los años recogidos (2012-2018) y en todas las matrices de distancia. Reseñar que para no hacer más extenso el informe pese a no consignarse en la tabla (3) los valores resultantes de las pruebas de permutaciones ( $Z$-value y $P$-value) todas las pruebas resultaron significativas y con valores $z$ suficientemente elevados.

Tabla 3: Resultados I de Moran TMD Años 2012-2018.

\begin{tabular}{|c|c|c|c|c|c|c|c|}
\hline Matriz de contigiuidad & $T M D 18$ & TMD17 & TMD16 & $T M D 15$ & $T M D 14$ & TMD13 & $T M D 12$ \\
\hline$Q 1$ & 0.509 & 0.516 & 0.535 & 0.518 & 0.552 & 0.553 & 0.503 \\
\hline$Q 2$ & 0.394 & 0.400 & 0.409 & 0.394 & 0.387 & 0.409 & 0.379 \\
\hline D1_6\%n & 0.454 & 0.469 & 0.481 & 0.468 & 0.468 & 0.490 & 0.454 \\
\hline D2_18/ $\mathrm{m}$ & 0.276 & 0.281 & 0.284 & 0.276 & 0.252 & 0.280 & 0.252 \\
\hline D3_36 km & 0.139 & 0.139 & 0.147 & 0.148 & 0.122 & 0.148 & 0.121 \\
\hline
\end{tabular}

Fuente: Elaboración propia con GeoDa. 
Siguiendo la línea que esbozan Acevedo-Blanco y Martínez-Quintana ${ }^{11}$ (2021) y en base a las proposiciones expuestas por Luc Anselin (2021) la interpretación de la DE global resulta conveniente extender su planteamiento en términos de incorporar la relación entre los valores pasados del indicador y el valor actual del indicador para cada una las ubicaciones concretas. Se propone de esta forma una especificación de modelos de regresión en las que los valores del indicador en el pasado predicen éstos en el futuro. Desde esta óptica espaciotemporal en el procedimiento reflejado en la tabla (4) se estandarizan variables y retardos tanto del año 2019 como del año 2012. De esta forma se procede a revisar una posible relación de dependencia global espaciotemporal de la TMD que confieran mayor robustez a los resultados hasta ahora examinados.

Tabla 4: Transformación de variables.

\begin{tabular}{|l|l|}
\hline Variable & Variable transformada \\
\hline TMD12 & TMD12st \\
\hline TMD19 & TMD19st \\
\hline Retardo TMD12 & Wtmd12st \\
\hline Retardo TMD19 & Wtmd19st \\
\hline
\end{tabular}

Fuente: Elaboración propia con GeoDa.

En el primero de los casos examinamos la relación entre una variable en dos momentos puntuales del tiempo ( $\mathrm{x}=\mathrm{TMD} 12 \mathrm{st} / \mathrm{y}=\mathrm{TMD} 19$ st.). Esta relación produce una pendiente muy significativa 0.765 sugiriendo una correlación temporal muy fuerte de la variable a lo largo del tiempo. En otras palabras, la pendiente del Gráfico (2) sugiere una fuerte correlación del paro a través del tiempo. Las ubicaciones con tasas altas de desempleo, y viceversa, presentan altas tasas de desempleo en eventos temporales posteriores. Esta correlación apunta al desempleo como elemento estructural en Andalucía. Municipios con alto nivel de desempleo continúan manteniendo las posiciones altas del ranking de paro casi una década después.

${ }^{11}$ Asociación Andaluza de Sociología. X Congreso Andaluz de Sociología. (Des)Poblaciones y Territorios. Una mirada hacia la periferia. Congreso Virtual en Línea. Baeza (Jaén) 21-23 de Enero 2021. 


\section{Gráfico 1: Diagrama de dispersión x=TMD12st / y=TMD19st.}

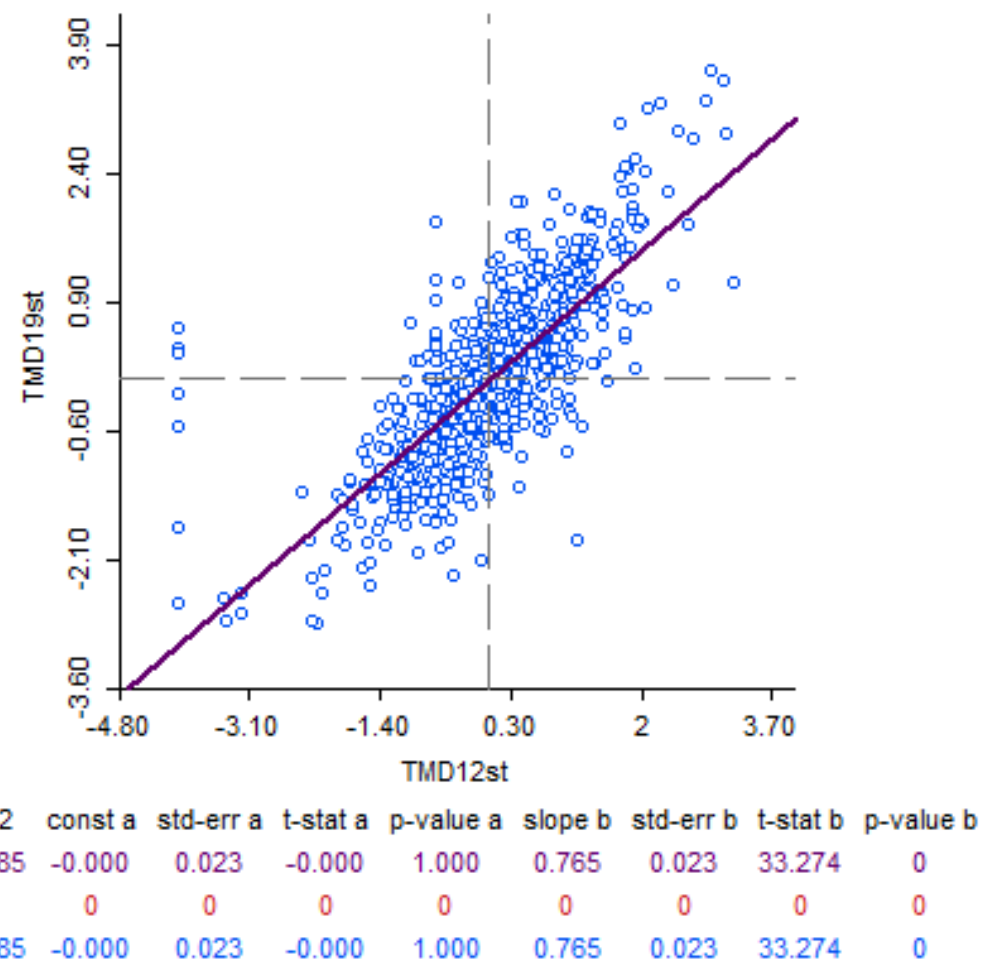

Chow test for selunsel regression subsets: need two valid regressions

Fuente: Elaboración propia con GeoDa

Es lógico, por otra parte, pensar que si existe una alta correlación de la variable entre diferentes momentos en el tiempo también exista una fuerte correlación temporal entre sus vecinos. Por tanto, la correlación entre retardos espaciales sugiere una alta correspondencia entre los dos periodos de tiempo revisados ( $\mathrm{x}=\mathrm{Wtmd12st} / \mathrm{y}=\mathrm{Wtmd19st}$. El Gráfico (2) nuevamente arroja una pendiente positiva con efectos de correlación muy elevados (0.902) incluso más altos que para la correlación de la variable en el tiempo examinada anteriormente. Esta relación sugiere acrecentar aún más las sospechas del carácter estructuralmente dependiente del contexto geográfico del desempleo en Andalucía. 


\section{Gráfico 2: Diagrama de dispersión $\mathrm{x}=\mathrm{Wtmd12st} / \mathrm{y}=\mathrm{Wtmd19st}$.}

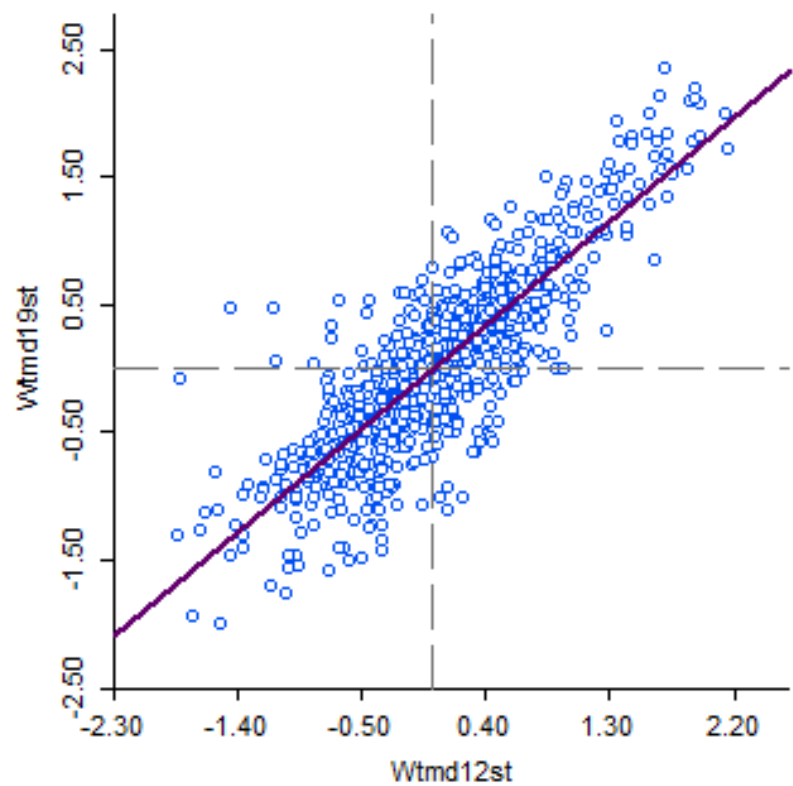

$\begin{array}{cccccccccc}\text { \#0bs } & \mathrm{R}^{\wedge} 2 & \text { const a } & \text { std-err a } & \text { t-stat a } & \text { p-value a } & \text { slope b } & \text { std-err b } & \text { t-stat b } & \text { p-value b } \\ 786 & 0.721 & -0.017 & 0.013 & -1.234 & 0.218 & 0.902 & 0.020 & 45.029 & 0 \\ 0 & 0 & 0 & 0 & 0 & 0 & 0 & 0 & 0 & 0 \\ 786 & 0.721 & -0.017 & 0.013 & -1.234 & 0.218 & 0.902 & 0.020 & 45.029 & 0\end{array}$

Chow test for selunsel regression subsets: need two valid regressions

Fuente: Elaboración propia con GeoDa

Por último, cabe reseñar que quizás la formulación más apropiada para una regresión espaciotemporal se expresa en torno a poner de manifiesto como los valores de los retardos espaciales pueden "difundirse" a las ubicaciones asociadas en periodos de tiempo posteriores ( $\mathrm{x}=\mathrm{Wtmd} 12 \mathrm{st} / \mathrm{y}=\mathrm{TMD} 19 \mathrm{st})$. O, en otros términos, los resultados parecen indicar la influencia que ejercen los vecinos en los niveles de desempleo municipal en Andalucía. La regresión sugiere que el valor de la TMD en cada ubicación correlaciona con los valores propios de los retardos espaciales en momentos anteriores en el tiempo. De nuevo el valor de la pendiente es bastante significativa (0.902) apuntándose en la interpretación de la regresión un enérgico efecto difusión del desempleo en Andalucía. 


\section{Gráfico 3: Gráfico 4 Diagrama de dispersión $x=W$ tmd12st / y=TMD19st.}

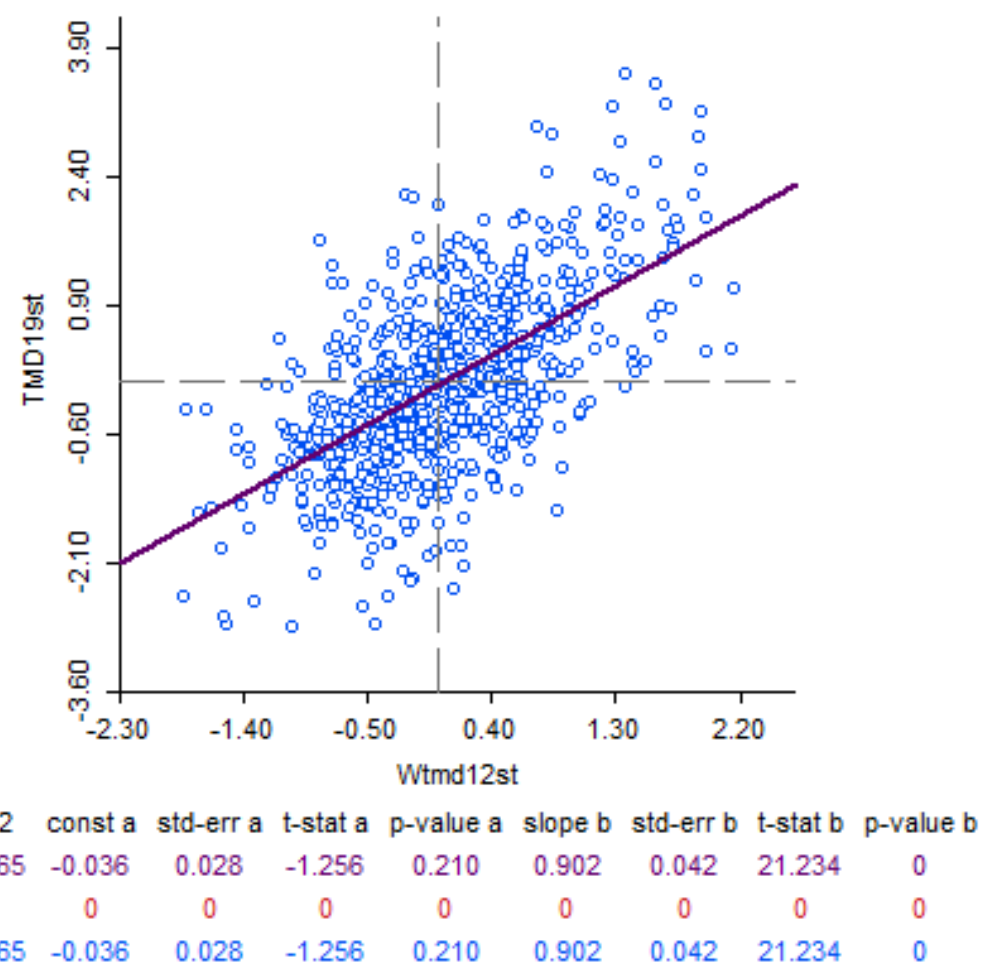

Chow test for selunsel regression subsets: need two valid regressions

\subsection{Dependencia local de la TMD. Mapas LISA.}

Para seguir profundizando en el análisis los resultados de los clústeres creados a partir del I local de Moran para cada una de las matrices de pesos espaciales desarrolladas se proyectan en el cuadro (3) de mapas LISA. El Mapa LISA proyecta las ubicaciones municipales que han resultado significativas en la prueba de permutación inferencial distribuidas en un esquema de cuatro colores por tipo de asociación. De esta forma se identifica los clústeres formados y los valores significativamente altos de la variable (alto-alto High/High) con el color rojo y los valores bajos (Low/Low bajo-bajo) con el color azul. Otros colores atenuados representan los clústeres compuestos por outliers o atípicos espaciales (alto-bajo y bajo alto). 
Al proponer cada una de las ubicaciones al enfoque de aporte para la construcción de la I de Moran el estadístico viene a evidenciar el grado de contribución de cada ubicación en la construcción del clúster. En otros términos, los clústeres informan ubicaciones cuyos valores han resultado significativamente positivos en la formación del índice $\mathrm{I}_{\mathrm{i}}$ mediante permutación inferencial en cada ubicación espacial. De este razonamiento se desprende que los clústeres formados no son auténticamente clústeres en sí mismos, sino que más bien se les podría denominar "núcleos de clústeres".

Los resultados del cuadro (3) evidencian la concentración del desempleo andaluz en los núcleos geográficos que el análisis exploratorio de la TMD revelaba. Cabe destacar que los resultados del mapa LISA de matrices de contigüidad simple (LISA 1 Q1) resultan similares a su análogo en cuanto a nivel para las matrices de distancias (D1 6Km). En otros términos, matriz de distancia para 6 kilómetros y contigüidad de primer orden reflejan iguales formaciones de clústeres para la TMD. Este resultado parece se vuelve a confirmar si realizamos igual comparación para el segundo grupo de LISA (LISA 2 Q2 / D2 18Km). En este sentido resaltar la evidencia de autocorrelación local del desempleo en Andalucía observándose la formación de clústeres similares al utilizar matrices de contigüidad de segundo orden, semejantes a MLT, y matrices de distancia entre centroides de 18 kilómetros, que podrían traducirse con 30 minutos de distancia laboral de desplazamientos. 


\section{Cuadro 3: Cuadro de Mapas LISA.}
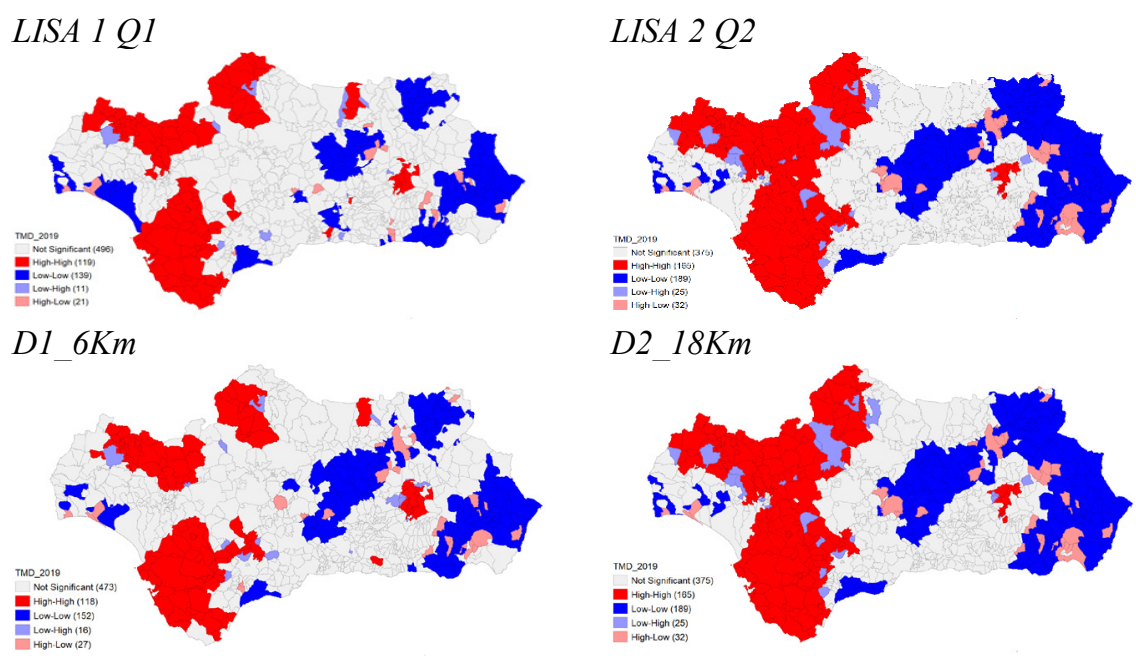

D2_ $18 \mathrm{Km}$
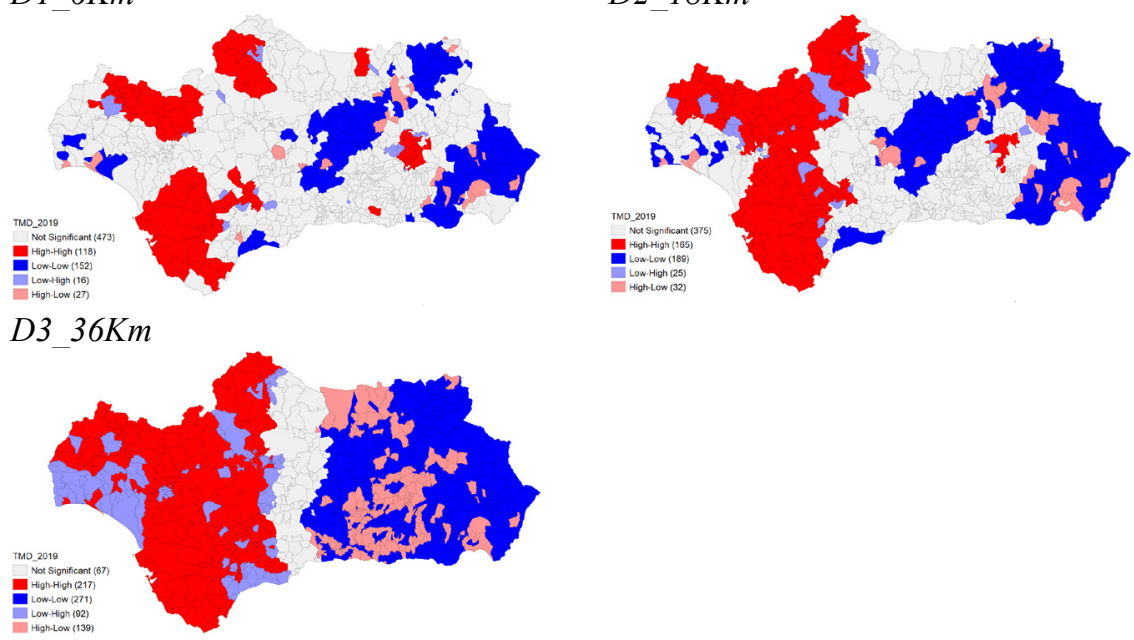

Fuente: Elaboración propia con GeoDa.

Especial consideración merece el mapa LISA construido a partir de la matriz de distancia más amplia (D3_36 Km). La distancia entre centroides de 36 kilómetros viene a reflejar aproximadamente una hora de desplazamiento laboral que, como se indicaba anteriormente, refleja una distancia de trayecto laboral máxima en la delimitación de los MTL. Los resultados que presentamos proyectados en el mapa (2) plantean una clara polarización en cuanto al desempleo entre la Andalucía oriental y occidental. En este sentido reseñar brevemente que dentro de esta polarización que divide Andalucía en dos mitades en cada una de estas partes se incluyen territorios internos con clústeres con comportamiento diferenciados en cuanto al indicador de desempleo.

Un análisis geográfico-económico exhaustivo traspasa los límites de este artículo exploratorio. Si bien para los clústeres de alta incidencia del desempleo (High-High) se propone un capítulo aparte, cabe reseñar sucintamente la existencia de clústeres de baja incidencia del paro agrupados en torno a MLT diferenciales, con tejidos productivos locales especializados y ubicados principalmente 
en las zonas del litoral de Huelva, la costa del Sol, costa y provincia de Almería y epicentros de la provincia de Jaén en la capital y Baza en Granada.

\section{Mapa 2: LISA PROYECTADO MATRIZ D3_36Km.}

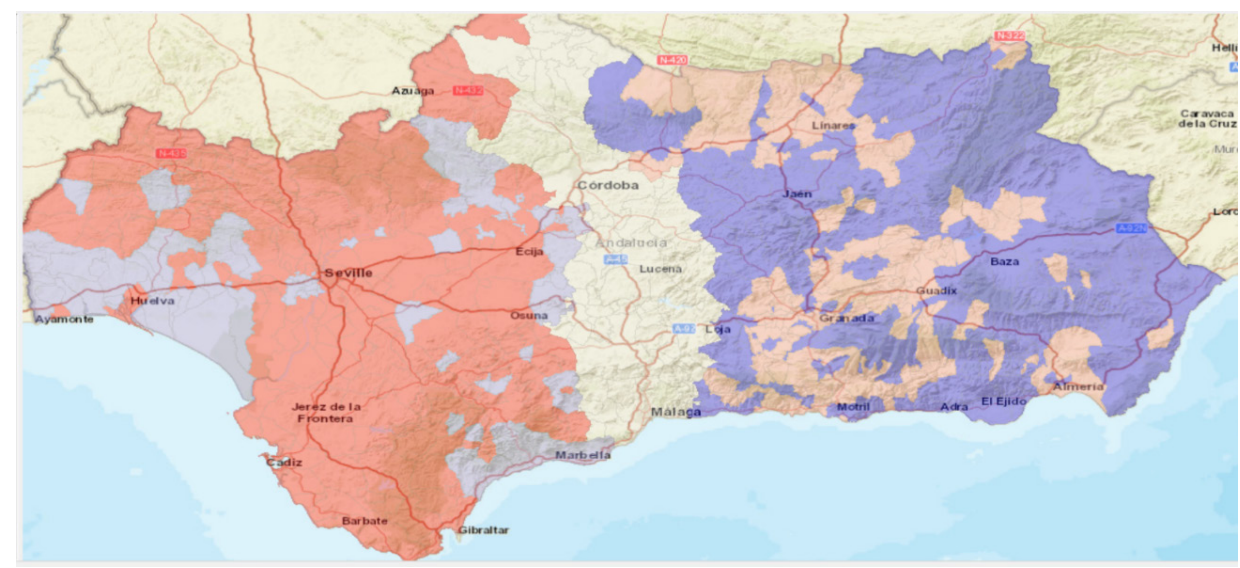

Fuente: Elaboración propia con GeoDa

\subsection{Clústeres de alta incidencia del desempleo en Andalucía.}

Para este último análisis de los datos y objetivo nuclear de este trabajo se utiliza en la localización de los clústeres de alta incidencia del desempleo en Andalucía el procedimiento más restrictivo; la ponderación de retardos con pesos espaciales de Reina (LISA 1 Q1). Se propone la ponderación espacial de contigüidad de primer orden como el más indicado para el examen geográfico y socioeconómico detallado por dos motivos fundamentales. Por una parte y desde una perspectiva estrictamente econométrica aunque resulte conveniente evaluar con varias ponderaciones de pesos espaciales, como apuntan Lesage y Pace (2014), un exceso de moldeado de matrices puede dar lugar a estimaciones sesgadas por los criterios de vecindad al incluirse en los modelos demasiados elementos de HE. Por otro lado, resulta lógico pensar que si el criterio de contiguiidad física de Reina resulta el mayormente desarrollado en casos de ESDA para análisis de variables socioeconómicas con unidades espaciales poligonales (Chasco y Fernández-Avilés 2009) apliquemos este marco de análisis para examinar los clústeres de alto desempleo en Andalucía. 


\section{Mapa 3: LISA1 Q1 PROYECTADO.}

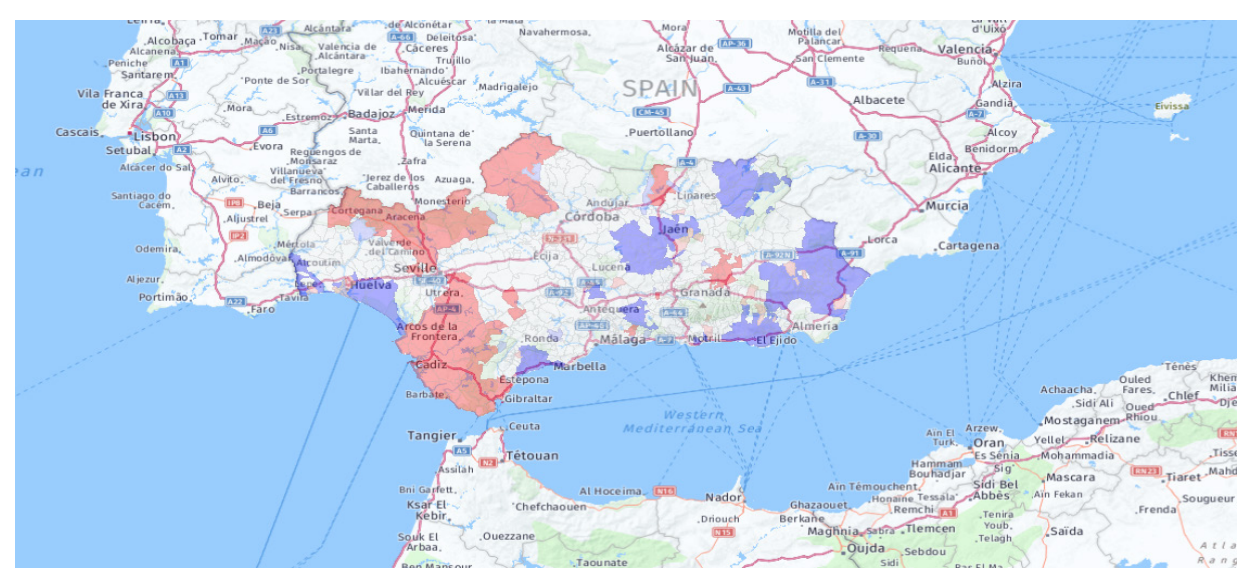

Fuente: Elaboración propia con GeoDa.

Si se parte de considerar que la media para todas las ubicaciones del $\mathrm{I}_{\mathrm{i}}$ local coincide con el I global, se puede afirmar que los valores atípicos superiores identificados en un diagrama de bisagras de Tukey corresponderán con las ubicaciones espaciales en las que la DE del indicador adquiere un comportamiento diferenciado del resto de valores. En otros términos, los valores de $\mathrm{I}_{\mathrm{i}}$ atípicos por su alto valor serán aquellos en los que se produce una alta concentración de DE positiva, tanto para valores altos de la variable, como para los valores más bajos.

En la Tabla (5) se muestran los valores atípicos superiores de la distribución de valores de I que han resultado a su vez clasificados en el cuadrante del mapa (3) correspondiente a la tipología High-High. Consecuentemente estos municipios pueden considerarse como los puntos realmente calientes (Hot spots) de la distribución por la alta contribución que tienen a la DE en la TMD. Simplificando los conceptos, los municipios que reflejan la tabla (5) identifican las ubicaciones más significativas y con mayor peso en la conformación de territorios de alta incidencia del desempleo producto del aporte de cada ubicación al índice I de Moran. 
Tabla 5: Hot Spots de I local de Moran.

\begin{tabular}{|c|c|c|c|c|}
\hline Alcalá de los Gazules & 11001 & Cádiz & 2,1978848 & $29,07 \%$ \\
\hline Algar & 11003 & Cádiz & 2,5095103 & $26,86 \%$ \\
\hline Arcos de la Frontera & 11006 & Cádiz & 3,6110568 & $34,86 \%$ \\
\hline Barbate & 11007 & Cádiz & 4,4959956 & $38,24 \%$ \\
\hline Benalup-Casas Viejas & 11901 & Cádiz & 1,8870006 & $26,36 \%$ \\
\hline Bornos & 11010 & Cádiz & 4,0037362 & $30,44 \%$ \\
\hline Cádiz & 11012 & Cádiz & 2,3749493 & $27,02 \%$ \\
\hline Chiclana de la Frontera & 11015 & Cádiz & 2,7910263 & $29,80 \%$ \\
\hline Chipiona & 11016 & Cádiz & 2,9823348 & $31,90 \%$ \\
\hline $\begin{array}{l}\text { El Puerto de Santa } \\
\text { María }\end{array}$ & 11027 & Cádiz & 2,135275 & $27,94 \%$ \\
\hline Espera & 11017 & Cádiz & 2,8992589 & $28,75 \%$ \\
\hline Jerez de la Frontera & 11020 & Cádiz & 3,0252439 & $30,38 \%$ \\
\hline Medina Sidonia & 11023 & Cádiz & 2,7600637 & $29,92 \%$ \\
\hline Paterna de Rivera & 11025 & Cádiz & 5,1500371 & $35,17 \%$ \\
\hline Prado del Rey & 11026 & Cádiz & 2,2359054 & $30,09 \%$ \\
\hline Puerto Real & 11028 & Cádiz & 2,5666891 & $28,11 \%$ \\
\hline San Fernando & 11031 & Cádiz & 2,8833668 & $30,53 \%$ \\
\hline San José del Valle & 11902 & Cádiz & 2,6509202 & $28,78 \%$ \\
\hline Sanlúcar de Barrameda & 11032 & Cádiz & 1,7774019 & $30,44 \%$ \\
\hline Vejer de la Frontera & 11039 & Cádiz & 3,4624373 & $31,83 \%$ \\
\hline Villamartín & 11041 & Cádiz & 2,5954471 & $30,15 \%$ \\
\hline Belmez & 14009 & Córdoba & 5,4065006 & $38,71 \%$ \\
\hline Fuente Obejuna & 14029 & Córdoba & 5,1388964 & $33,63 \%$ \\
\hline Hinojosa del Duque & 14035 & Córdoba & 2,5434654 & $28,06 \%$ \\
\hline La Granjuela & 14032 & Córdoba & 6,6934375 & $36,54 \%$ \\
\hline Los Bláquez & 14011 & Córdoba & 5,4429246 & $33,18 \%$ \\
\hline $\begin{array}{l}\text { Peñarroya- } \\
\text { Pueblonuevo }\end{array}$ & 14052 & Córdoba & 6,9615806 & $36,98 \%$ \\
\hline Valsequillo & 14064 & Córdoba & 2,0465109 & $25,26 \%$ \\
\hline Villanueva del Rey & 14071 & Córdoba & 2,0236731 & $27,18 \%$ \\
\hline Alájar & 21001 & Huelva & 2,1146049 & $32,99 \%$ \\
\hline Arroyomolinos de León & 21009 & Huelva & 3,6959535 & $32,70 \%$ \\
\hline Cala & 21016 & Huelva & 4,3586688 & $35,27 \%$ \\
\hline Cañaveral de León & 21020 & Huelva & 1,7268827 & $26,82 \%$ \\
\hline Castaño del Robledo & 21022 & Huelva & 3,1301801 & $31,24 \%$ \\
\hline Galaroza & 21034 & Huelva & 1,6452992 & $27,22 \%$ \\
\hline Los Marines & 21048 & Huelva & 2,1685184 & $30,98 \%$ \\
\hline El Cuervo de Sevilla & 41903 & Sevilla & 2,1125474 & $28,42 \%$ \\
\hline $\begin{array}{l}\text { Las Cabezas de San } \\
\text { Juan }\end{array}$ & 41020 & Sevilla & 2,4092231 & $29,39 \%$ \\
\hline San Nicolás del Puerto & 41088 & Sevilla & 1,7501691 & $29,29 \%$ \\
\hline Utrera & 41095 & Sevilla & 2,1420731 & $30,00 \%$ \\
\hline
\end{tabular}


Se utiliza para representar la tabla (3) un mapa de Co - localización (Mapa 4) en el que el esquema de la leyenda representa valor 0 para los municipios no significativos en el cálculo del estadístico local $\left(\mathrm{I}_{\mathrm{i}}\right)$. Por su parte el valor de la leyenda Others hace referencia a los municipios que resultando significativos en $\mathrm{I}_{\mathrm{i}}$ no han resultado catalogados en el clúster High-High del LISA. Por último, el valor 1 en la leyenda representan a los municipios clasificados simultáneamente en el referido clúster High-High del LISA y en la distribución de atípicos de $\mathrm{I}_{\mathrm{i}}$. cuyos valores de TMD son especialmente altos.

\section{Mapa 4: Hot spots de I local de Moran Tabla (3).}

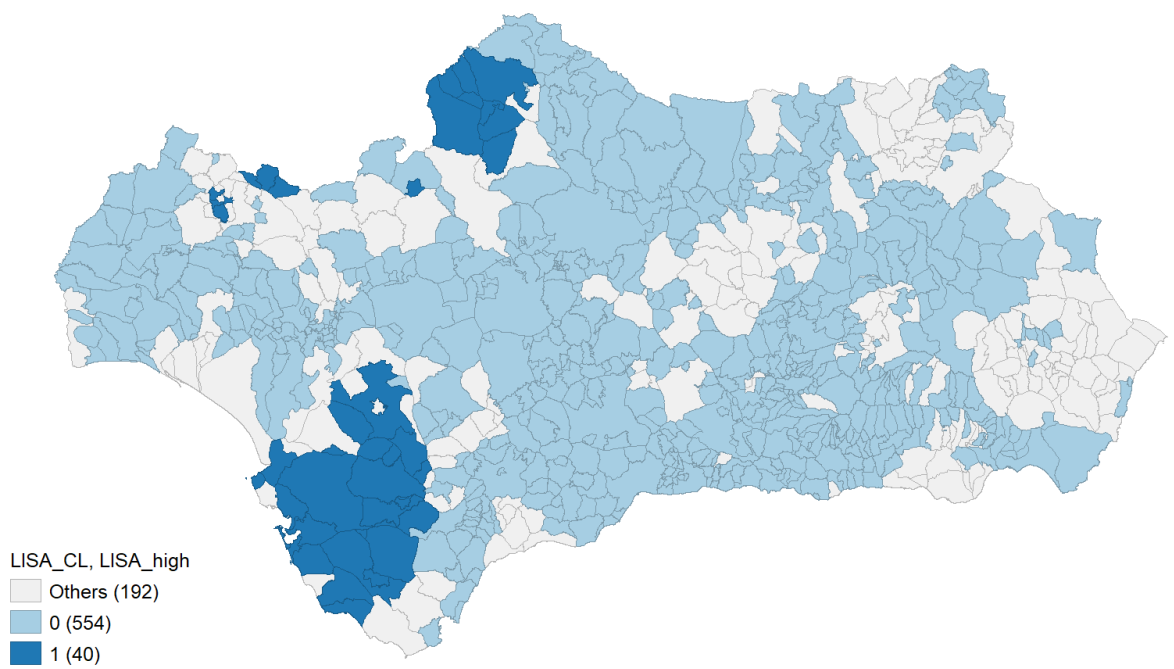

Fuente: Elaboración Propia con GeoDa.

En primer lugar, comenzaremos por la agrupación más al norte localizada en la provincia de Córdoba. Los ocho municipios que han resultado positivos en el análisis coinciden en formar parte de la Comarca del Guadiato ${ }^{12}$. El valor medio de TMD de esta agrupación de municipios se sitúa aproximadamente en $33.65 \%$ sumando 4104 desempleados y 8090 afiliaciones de media anual en 2019. Los datos de desempleo de este clúster hablan por sí solos de la depresión socioeconómica de la Comarca.

Por su parte seis son los municipios que forman el clúster perteneciente en su mayor parte a la comarca de la Sierra Norte ${ }^{13}$ de Huelva. Este clúster con un valor medio de TMD situado en 33.60\%. Integran el conglomerado 491 desempleados con 973 afiliaciones de media anual para 2019. Cabe destacar que

${ }^{12}$ Valle del Guadiato, Wikipedia https://es.wikipedia.org/wiki/Valle del Guadiato\#Municipios

${ }^{13}$ Comarca de la Sierra Norte de Huelva https://es.wikipedia.org/wiki/Sierra de Huelva 
este territorio cuenta con un importante atractivo turístico además de un valioso patrimonio medioambiental y paisajístico con gran potencial para un desarrollo sostenible no traducido en oportunidades de empleo para la comarca.

En orden a la división territorial con la que estamos trabajando apuntar que la provincia de Sevilla cuenta con tres municipios más al sur ubicados (Utrera, Las Cabezas de San Juan y El Cuervo) entre los Hot spots de desempleo resultantes del análisis. Situados ${ }^{14}$ en las Comarcas de La Campiña, de la que Utrera es cabeza de partido judicial, y la Comarca de Las Marismas del Bajo Guadalquivir, estos municipios cuentan con una TMD que oscila de media sobre el $29.27 \%$ de desempleo. En contraste con las comarcas que anteriormente revisamos estos territorios cuentan con una amplia densidad de población sumando entre los tres municipios 27442 afiliaciones y 11579 personas registradas como demandantes de empleo (DENOS). Por otra parte, el municipio de San Nicolás del Puerto, situado a 95 kilómetros de la capital, aunque perteneciente a la provincia de Sevilla, podría clasificarse más bien junto a los municipios de la Sierra Norte de Huelva en base a su proximidad geográfica y similitud de características sociodemográficas.

El caso de la provincia de Cádiz merece una especial atención y un estudio más detallado que por las limitaciones propias del espacio no podemos abarcar. En la tipología clasificatoria que desarrollamos (tabla 5) 22 de los 40 municipios que integran el clúster pertenecen a la provincia de Cádiz. 112884 DENOS y 263856 afiliaciones a la Seguridad Social con una media de TMD del orden del $30 \%$ reflejan la situación de desempleo masivo y estructural de la provincia. Entre los municipios que integran este clúster encontramos poblaciones con gran densidad de población como Jerez de la Frontera o la capital administrativa de la provincia, Cádiz. El mapa (5) muestra con fondo de tonos rosáceos los positivos en el clúster municipios y con fondo claro de color amarillo los no clasificados. Que casi la mitad de los municipios gaditanos (22 de 45) se encuentran clasificados en el clúster nos lleva a advertir de la gran problemática social y económica que para la provincia de Cádiz supone este desempleo masivo.

${ }^{14}$ Comarcas de la Campiña y Marismas del Bajo Guadalquivir: https://es.wikipedia.org/wiki/ Bajo Guadalquivir (comarca) https://es.wikipedia.org/wiki/La Campi\%C3\%B1a (Sevilla) 


\section{Mapa 5: Provincia de Cádiz clasificación de Hot Spots tabla (3)}

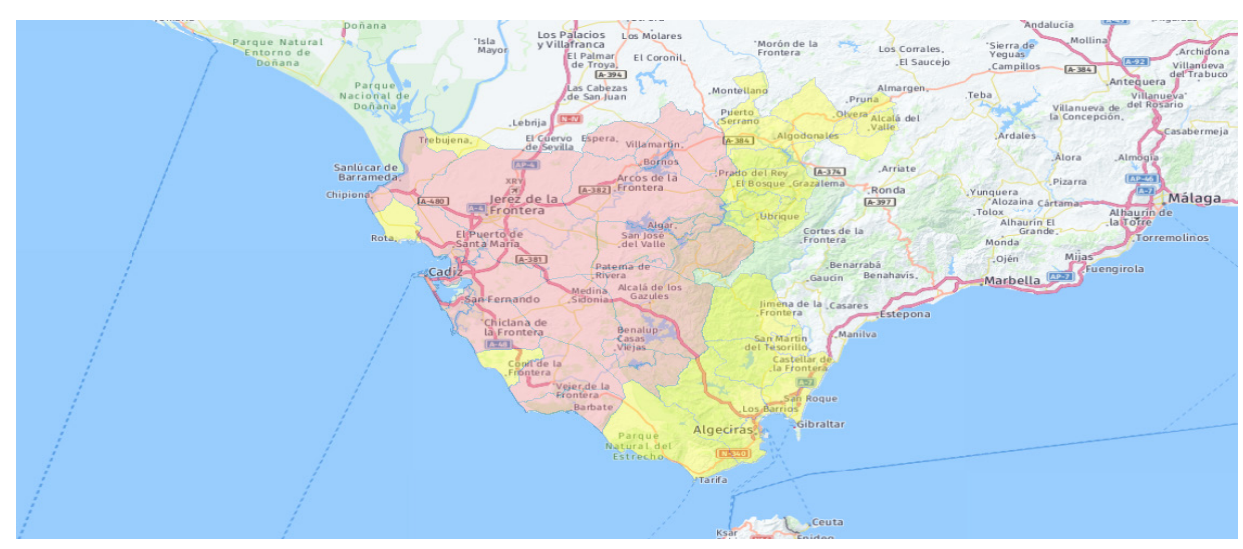

Fuente: Elaboración Propia con GeoDa.

\section{CONCLUSIONES}

Siguiendo a (Sanchís-Gómez, 2003:170) con todas la cautelas podemos afirmar que "en España el interés de la Sociología por la problemática del desempleo no parece abrumadora". Han sido disciplinas, que en gran medida cuentan con un campo y objeto de estudios paralelos a la explicación sociológica, como la Psicología, la Psiquiatría o la Psicología Social las que han focalizado más su atención en las experiencias del desempleo.

Parece que las consecuencias del desempleo a nivel territorial hayan quedado subsumidas bajo el paraguas del discurso social generalizado del paro asentado la estigmatización del desempleado y su hábitat comunitario. La percepción psicosocial extendida hacia estos colectivos, y los territorios en los que habitan, se basa en asignarles a los agregados de parados toda una carga estigmatizadora de déficits individuales e indolencia producto de las cuales ven rebajada sustancialmente su capacidad de empleabilidad e inclusión sociolaboral (Escarbajal-Frutos y Izquierdo-Rus, 2013). Y es que la noción de parado ya desde los orígenes etimológicos de la palabra contiene tanto en lengua inglesa como en francesa una componente despectiva e irónica que denota inactividad, falta de interés, apatía e indolencia (Plugiese, 2000). Desde esta óptica Prieto (2007) argumenta que la norma socialmente construida es el trabajo, ocupando al mismo tiempo un lugar central en las sociedades laborales contemporáneas. Por tanto, transgredir lo normativo conlleva inexorablemente revitalizar la carga social de indolencia y falta de interés por conseguir un empleo que el concepto de parado ya de por sí lleva aparejado.

Este trabajo se ha centrado en proponer un ejercicio ESDA aplicado al estudio del paro en Andalucía. El artículo basa su analítica en los supuestos del 
paradigma y la visión Geo-Sociológica en la que el territorio, representado por el retardo espacial, se plantea a modo de variable explicativa en correlación de situaciones de desempleo entre unidades espaciales contiguas (Acevedo-Blanco, 2019). En orden a los objetivos analíticos planteados y a los resultados obtenidos se desprende que no solo prevalecen variables individuales en el fenómeno social del desempleo. La explicación sociológica en aras de poder establecer diagnósticos precisos también ha de considerar el territorio en el que residen las personas como una variable de análisis más entre las explicativas, desencadenantes y condicionantes de las situaciones de desempleo.

De los resultados de este trabajo se desprende un contexto de autocorrelación espacial global del paro en Andalucía del cual podemos extraer las principales evidencias:

i. Globalmente, a partir de los índices de I de Moran positivos, se puede afirmar que los municipios en Andalucía están rodeados de municipio contiguos con similares valores en la TMD, sean estos valores altos o bajos. Mediante el esquema de inferencia estadística basadas en permutaciones el procedimiento ha verificado la DE del desempleo en Andalucía.

ii. Los resultados del análisis de sensibilidad modificando la forma de conceptualización de la distancia añaden mayor robustez a los planteamientos pues confirman la autocorrelación del indicador para cada una de las matrices de pesos espaciales desarrolladas. En otras palabras, el análisis espacial revela una estructura en Andalucía de diferentes territorios heterogéneos de incidencia desempleo dependientes del contexto geográfico en el que se enclavan.

iii. Se evidencia una notable dependencia espaciotemporal del desempleo en Andalucía tanto si atendemos a la relación entre indicador en momentos anteriores en el tiempo, como si prestamos atención a las relaciones entre retardos en momentos temporales diferentes, o si se focaliza el análisis en el posible efecto difusión pudiendo llegar a condicionar los valores de los retardos espaciales en el pasado a los valores de la TMD en el presente. Estos resultados inciden de forma considerable en poner de relieve el carácter estructuralmente dependiente del contexto geográfico del desempleo andaluz.

Con respecto a la DE local para diferenciar los territorios sobre los que se conforma una mayor incidencia en la formación del indicador global de DE del paro se ha aplicado el estadístico I local de Moran. A partir de la distribución de índices locales de Moran para cada ubicación los resultados se han depurado seleccionando los atípicos superiores clasificados a su vez al clúster de valores altos del mapa (3) LISA (High-High). El procedimiento ha dado como resultado la tabla (5) mapeada (Mapa 4) en el que se reflejan los municipios andaluces con mayor significatividad positiva en la construcción del local de Moran, o expresado, en otros términos, los municipios andaluces que por su alta contribución en el índice global de DE conforman territorios espacialmente dependientes de 
un contexto de gran incidencia del desempleo. De estos resultados cabe destacar la singularidad del caso de la provincia de Cádiz en la que hemos podido comprobar como la casi totalidad de la provincia sufre condiciones masivas de desempleo.

Conforme al espíritu metodológico de esta publicación se han presentado las bases, el desarrollo y los principales procedimientos de una técnica que cada vez en mayor medida se abre paso entre las metodologías de análisis empírico en sociología económica. La metodología ESDA, más que conclusiones fuertes, pone de relieve una técnica de gran valor analítico exploratorio a la vez que práctico y aplicado. El objetivo en cuanto al estudio sociológico del desempleo tiene como misión ineludible contribuir con el análisis científico a ayudar a los poderes públicos en el propósito de realizar una mejor planificación local. En este sentido cabe destacar que para revelar las contingencias propias de los fenómenos sociales cada vez en mayor medida se recurre a herramientas de análisis capaces de orientar sus metodologías, técnicas y procedimientos en la toma de decisiones razonadas contando con la explotación de grandes bases de datos; también geográficas.

\section{BIBLIOGRAFÍA}

Acevedo-Blanco, A.J. (2021), "VAL_tmd_Empiria”, Mendeley Data, V1, doi: 10.17632/ g5 g6gk9 $9 \mathrm{~cm} .1$

Acevedo-Blanco, A. J. (2019). ESDA con software GEODA. El territorio como variable explicativa en el análisis sociológico. En Estrella Gualda Caballero (Ed.), Sociedades y Fronteras. Actas del IX Congreso Andaluz de Sociología (Collectane, pp. 543-569). UHU.ES PUBLICACIONES. Servicio de Publicaciones de la Universidad de Huelva.

Acevedo-Blanco, A.-J., y Martínez-Quintana, V. (2021). Autocorrelación espacial bivariante entre eventos temporales. Conceptos y aplicación mediante un ejercicio práctico del desempleo municipal en Andalucía. X Congreso Andaluz de Sociología. Recuperado de: https://xcongresoandaluzdesociologia.ujaen.es/programa/

Acevedo-Bohórquez, I., y Velásquez, C. E. (2008). Algunos conceptos de la econometría espacial y el análisis exploratorio de datos espaciales. Ecos de Economía: A Latin American Journal of Applied Economics, 12(27), 9-2.

Agnew, J. (1996). Mapping politics: how context counts in electoral geography. Political Geography, 15(2), 129-146. https://doi.org/10.1016/0962-6298(95)00076-3

Albert, C., y Toharia, L. (2007). "Las estadísticas administrativas como fuentes de información para el estudio del mercado de trabajo andaluz." https://www.juntadeandalucia.es/institutodeestadisticaycartografia/estudios/administrativas/administrativas. pdf

Almazán, A., Arribas, J.-M., Camarero, L., y Vallejos, A.-F. (2011). Análisis estadístico para la investigación social. Garceta.

Alonso-Villar, O., Del-Río, C., y Toharia, L. (2009). Un análisis espacial del desempleo por municipios. Revista de Economía Aplicada, 17(49), 47-80.

Alonso, L. E., Rodríguez, C. J. F., y Rojo, R. I. (2017). Juventud y percepciones de la crisis: Precarización laboral, clases medias y nueva política. Empiria. Revista de 
Metodología de Ciencias Sociales, 37, 155-178. https://doi.org/10.5944/empiria.37.2017.18983

Anselin, L. (1995). Local Indicators of Spatial Association-LISA. Geographical Analysis, 27(2), 93-115. https://doi.org/10.1111/j.1538-4632.1995.tb00338.x

Anselin, L. (1999). The future of spatial analysis in the social sciences. Geographic Information Sciences, 5(2), 67-76. https://doi.org/10.1080/10824009909480516

Anselin, L. (2007). Spatial econometrics in RSUE: Retrospect and prospect. Regional Science and Urban Economics, 37(4), 450-456. https://doi.org/10.1016/j.regsciurbeco.2006.11.009

Anselin, L. (2021). Global Spatial Autocorrelation (2). Bivariate, Differential and EB Rate Moran Scatter Plot. Geoda WorkBook. https://geodacenter.github.io/ workbook/5b_global_adv/lab5b.html

Anselin, L., y Bera, K. (1998). Spatial dependence in linear regression models with an introduction to spatial econometrics. Statistics Textbooks and Monographs, 155, 237-290. https://doi.org/10.2307/1271490

Anselin, L., y Rey, S. (1991). Properties of Tests for Spatial Dependence in Linear Regression Models. Geographical Analysis, 23(2), 112-131. https://doi. org/10.1111/j.1538-4632.1991.tb00228.x

Celemín, J. P. (2009). Autocorrelación espacial e indicadores locales de asociación espacial. Importancia, estructura y aplicación. Revista Universitaria de Geografía, 18(1), $11-31$.

Chasco, C. (2003). Econometría Espacial Aplicada a la Predicción-Extrapolación de Datos Microterritoriales. Dirección General de Economía y Planificación. Comunidad de Madrid. http://www.revista-eea.net/documentos/22326.pdf

Chasco, C. (2004). Modelos de Heterogeneidad Espacial. EconPapers. University Library of Munich, Germany.

Chasco, C., y Fernández-Avilés, G. (2009). Análisis de datos espacio-temporales para la economía y el geomarketing. Netbiblo.

Dall'erba, S. (2009). Exploratory Spatial Data Analysis. International Encyclopedia of Human Geography, 683-690. https://doi.org/10.1016/B978-008044910-4.00433-8

Durkheim, E. (1992). El suicidio (Vol. 37). Ediciones Akal.

Escarbajal-Frutos, A., y Izquierdo-Rus, T. (2013). Percepciones psicosociales de la exclusión que determinan la inclusión sociolaboral. Revista de Ciencias Sociales, 19(1), 13-21.

Gómez-Garrido, M. (2005). Dos formas distintas de investigar el problema del paro en los albores de la encuesta social. El juicio público frente a la taxonomía. Empiria. Revista de Metodología de Ciencias Sociales, O(9), 115. https://doi.org/10.5944/ empiria.9.2005.1006

Gutiérrez-Puebla, J., y García-Palomares, J. (2016). Big (Geo)Data en Ciencias Sociales: Retos y Oportunidades. Revista de Estudios Andaluces, 33(331), 1-23.

Jahoda, M. (1987). Empleo y desempleo: un análisis socio-psicológico. Ediciones Morata.

Kosfeld, R, y Dreger, C. (2006). Thresholds for Employment and Unemployment - a Spatial Analysis of German Regional Labour Markets 1992-2000. Papers in Regional Science, 85, 523-542. https://doi.org/https://doi. org/10.1111/j.1435-5957.2006.00096.x

Kosfeld, Reinhold, y Dreger, C. (2019). Towards an East German wage curve - NUTS boundaries, labour market regions and unemployment spillovers. Regional Science 
and Urban Economics, 76, 115-124. https://doi.org/https://doi.org/10.1016/j.regsciurbeco.2018.01.006

Lesage, J. P., y Pace, R. K. (2014). The biggest myth in spatial econometrics. Econometrics, 2(4), 217-249. https://doi.org/10.3390/econometrics2040217

Li, X., Anselin, L., y Koschinsky, J. (2015). GeoDa web. Proceedings of the 23rd SIGSPATIAL International Conference on Advances in Geographic Information Systems - GIS '15, November, 1-4. https://doi.org/10.1145/2820783.2820792

López-Bazo, E., Del-Barrio, T., y Artis, M. (2002). The regional distribution of spanish unemployment. A spatial analysis. Papers in Regional Science, 81, 365-389. https:// doi.org/https://doi.org/10.1111/j.1435-5597.2002.tb01239.x

López-Bazo, E., Del-Barrio, T., y Artís, M. (2005). The Geographical Distribution Of Unemployment In Spain. Regional Studies-, 39(3), 305-318.

López-Hernández, F. A. (2013). Second-order polynomial spatial error model. Global and local spatial dependence in unemployment in Andalusia. Economic Modelling, 33, 270-279. https://doi.org/10.1016/j.econmod.2013.04.014

Manzanares-Gutiérrez, Á., y Riquelme-Perea, P. (2017). Análise espacial do desemprego nos mercados locais de traballo españois. Revista Galega de Economía: Publicación Interdisciplinar Da Facultade de Ciencias Económicas e Empresariais, 26(2), 29_ 42.

Manzanares-Gutiérrez, Á., Sachez-López, C., y Riquelme-Perea, P. (2016). Análisis de la coherencia en los mercados locales de trabajo de la provincia de Huelva. Revista de Estudios Regionales, 107, 177-205.

Martínez-Quintana, V. (2004). Desempleados adultos de larga duración en Tendencias en Desigualdad y exclusión social. In J. F. Tezanos (Ed.), Tendencias en Desigualdad y exclusión social. Tercer Foro sobre Tendencias Sociales. (Sistema, pp. 253295). Sistema.

Martínez-Quintana, V. (2011). Los parados de larga duración: situaciones y perspectivas ante el desempleo y su pobreza. Temas Para El Debate, 205, 35-38.

Martínez-Tola, E., y Cal-de-la, M. L. (2017). Una aproximación territorial y de género al desempleo durante la crisis. el caso de los municipios vascos. Lurralde: Investigación y Espacio., 40, 247-272.

Mills, C. W. (1977). La imaginación sociológica. México: Fondo de Cultura Económica.

Möller, J., y Soltwedel, R. (2007). Recent developments of regional labor market analysis using spatial econometrics: Introduction. International Regional Science Review, 30(2), 95-99. https://doi.org/10.1177/0160017606298420

Moran, P. (1950). Notes on Continuous Stochastic Phenomena. Biometrika, 37(1/2), 17-23. http://www.jstor.org/stable/2332142

Netrdová, P., y Nosek, V. (2016). Spatial patterns of unemployment in Central Europe: emerging development axes beyond the Blue Banana. Journal of Maps, 12(4), 701-706. https://doi.org/10.1080/17445647.2015.1063467

Netrdová, P., y Nosek, V. (2020). Spatial dimension of unemployment: Space-time analysis using real-time accessibility in Czechia. ISPRS International Journal of Geo-Information, 9(6). https://doi.org/10.3390/ijgi9060401

Patacchini, E., y Zenou, Y. (2007). Spatial dependence in local unemployment rates. Journal of Economic Geography, 7(2), 169-191. https://doi.org/10.1093/jeg/lbm001

Plugiese, E. (2000). Qué es el desempleo. Política y Sociedad, 34, 59-67.

Porter, J. R. (2011). Context, Location, and Space: The Continued Development of our "Geo-Sociological" Imaginations. The American Sociologist, 42(4), 288-302. https://doi.org///dx.doi.org.ezproxy.uned.es/10.1007/s12108-011-9131-4 
Poveda-Rosa, M. (2006). "Los lunes al sol” o "los lunes en casa": Roles de género y vivencias del tiempo de desempleo. Cuadernos de Relaciones Laborales, 24(2), 85110. https://doi.org/10.5209/CRLA.33456

Prieto, C. (2007). Del estudio del empleo como norma social al de la sociedad como orden social. Papeles Del CEIC: International Journal on Collective Identity Research, 2007(1), 0.

Prodromídis, P. I. K. (2012). Modeling male and female employment policy in Greece from local data. Economic Modelling, 29(3), 823-839. https://doi.org/10.1016/j. econmod.2011.09.004

Rodrigues-Silveira, R. (2013). Representación espacial y mapas. Centro de Investigaciones Sociológicas (CIS) Cuadernos Metodológicos, Vol. 50.

Rodríguez Moya, J. M., y Pozo Rivera, E. (2019). Análisis territorial del desempleo por género en la Comunidad de Madrid durante la crisis (2008-2014). Cuadernos Geográficos, 58(1), 277-299. https://doi.org/10.30827/cuadgeo.v58i1.5630

Sanchis-Gómez, E. (2017). Los parados: cómo viven, qué piensan, por qué no protestan. Universitat de València.

Sanchís-Gómez, E. (2003). La experiencia de paro. Política y Sociedad, 40(1), 161-184.

Santos-Ortega, A. (2004). Trayectorias sociales del paro de larga duración. Universidad Complutense de Madrid. Tesis Doctoral

Soja, E. (1980). The Socio-Spatial Dialectic. Annals of the Association of American Geographers, 70(2), 207-225.

Stamm, M. (2006). Desempleo juvenil. Perfiles de una reflexión psicosocial. Revista Europea de Formación Profesional, 39, 113-123.

Stephan, F. F. (1934). Sampling Errors and Interpretations of Social Data Ordered in Time and Space. Journal of the American Statistical Association, 29(185A), 165166. https://doi.org/1The Moran Scatterplot as an ESDA Tool to Assess Local Instability in Spatial Association.

Suárez, P., Mayor, M., y Cueto, B. (2012). The accessibility to employment offices in the Spanish labour market. Papers in Regional Science, 91(4), 823-848. https://doi. org/10.1111/j.1435-5957.2012.00425.x

Sui, D. Z. (2004). Tobler's First Law of Geography : Annals of the Association of American Geographers, 94(2), 269-277. https://doi. org/10.1111/j.1467-8306.2004.09402003.x

Therborn, G. (2006). Why and How Place Matters. In The Oxford Handbook of Contextual Political Analysis. Oxford University Press. https://doi.org/10.1093/oxford$\mathrm{hb} / 9780199270439.003 .0027$

Tobler, W. R. (1970). A Computer Movie Simulation Urban Growth in Detroit Region. Economic Geography, 46, 234-240. https://doi.org/10.1126/science.11.277.620

Vilalta, C. J. V (2005). Cómo enseñar autocorrelación espacial. Economía, Sociedad y Territorio, v(18), 323-333. http://www.redalyc.org/articulo.oa?id=11101804 
